\title{
La comunicación entre el personal sanitario y los afectados por una amputación traumática. La sábana por encima
}

\author{
José Luís Díaz Agea \\ César Leal Costa \\ Paloma Echevarría Pérez \\ Universidad Católica San Antonio \\ (Murcia, España) \\ María Rosario Martín Robles \\ Servicio Murciano de Salud \\ (Murcia, España)
}

\section{Palabra clave \\ amputación traumática accidentes información comunicación estigma}

\begin{abstract}
Resumen
En este trabajo se presentan parte de los resultados de una investigación cualitativa de tipo etnográfico centrada en el análisis de los discursos de personas amputadas por accidente de moto en la región de Murcia (España). Concretamente los referidos a la esfera de la comunicación entre el personal sanitario y los afectados por una amputación traumática en los momentos que rodean el acto quirúrgico o la pérdida del miembro en el accidente. Como objetivos planteamos conocer la valoración subjetiva de la atención recibida durante el ingreso hospitalario y analizar las opiniones en torno a la información ofrecida a los recién amputados por causa traumática. La metodología que se llevó a cabo para aproximarnos al fenómeno humano de la comunicación y la interacción en un contexto experiencial de sufrimiento y dolor fue la etnografía utilizando técnicas de investigación como la entrevista semiestructurada, la historia de vida y la observación participante. Sometimos posteriormente los discursos a un análisis de contenido con orientación hermenéutica abarcando el contexto en el que se producen dichos testimonios. Los resultados, siempre desde la subjetividad de los afectados, dan cuenta de que en ocasiones la información sobre el proceso que se experimenta en una amputación traumática no ha sido clara y ha estado mal administrada, lo que redundaría en al aumento del sufrimiento de estas personas. Estando la cuestión de la mutilación del cuerpo rodeada todavía de un halo de estigmatización que dificulta el apoyo prestado.
\end{abstract}




\section{Communication between Health Workers and Patients Affected by Traumatic Amputation. The Sheet Over}

\begin{tabular}{c}
\hline Keywords \\
\hline traumatic amputation \\
accidents \\
information \\
communication \\
stigma
\end{tabular}

\section{Cómo citar el artículo}

Díaz Agea, J. L.; Leal Costa, C.; Martín Robles, M. R. y Echevarría Pérez, P. (2013). La comunicación entre el personal sanitario y los afectados por una amputación traumática. La sábana por encima. Revista de Comunicación y Salud, 3(2), pp. 5-19.

DOI: http://doi.org/10.35669/revistadecomunicacionysalud.2013.3(2).5-19

\begin{abstract}
The present article presents some of the results of an ethnographic qualitative study focused on the discourse analysis of motorcycle accident amputees in the Region of Murcia (Spain). Particularly those related to the field of communication between health workers and the affected by traumatic amputation in the moments surrounding the surgery or limb loss in the accident. As objectives we set out to meet the subjective evaluation of the care provided during hospitalization and analyze the opinions about the information given to new amputees due to a traumatic cause. The methodology employed to approximate the human phenomenon of communication and interaction in an experiential context of suffering and pain was ethnography research using techniques such as semi-structured interviews, life history and participant observation. Subsequently we submitted speeches to a content analysis with hermeneutic orientation covering the context in which these statements occur. The results, provided the subjectivity of the affected, reveal that sometimes the information about the process that is experienced in a traumatic amputation was not clear and has been poorly managed, possibly leading to increased suffering of these people. While the issue of body mutilation still surrounded by a halo of stigma that hinders the support.
\end{abstract}




\section{Introducción.}

En las últimas décadas el modelo de relación entre los profesionales sanitarios y el paciente está sufriendo una profunda transformación (Lázaro y Gracia, 2006). El poder y el control en la relación, hasta ahora mayoritariamente en manos de los profesionales, tienden en la actualidad a ser compartidos con el paciente, promoviéndose así la participación del paciente en el proceso de salud-enfermedad (Mead y Bower, 2000). Los pacientes están adquiriendo progresivamente un papel más activo, siendo más conscientes de sus derechos y responsabilidades, que son amparados por la ley, como la Ley de Autonomía del Paciente (2002) en España, promovidos por organismos internacionales como la Organización Mundial de la Salud en la obra Doctor Patient Interaction and Communication (1993) y por proveedores de salud como el National Health Service británico (General Medical Council, 2001).

Esta orientación centrada en el paciente es extraña en la relación clásica médicopaciente, que se ha sustentado tradicionalmente en el paternalismo. La relación paternalista en la asistencia sanitaria busca procurar el mayor bien al paciente, siendo definido tal bien, únicamente, por los profesionales sanitarios. Sin embargo, en la actualidad, las sociedades occidentales han ido avanzando hacia relaciones sociales democráticas presididas por la idea del consentimiento libre e informado de los ciudadanos. La actual orientación "al cliente" de los servicios públicos es fruto de este cambio de mentalidad (Simón, 2000).

Investigaciones empíricas realizadas en muy distintos contextos culturales (Brédart, Bouleuc, y Dolbeault, 2005; Mead y Bower, 2000; Mira y Aranaz, 2000; Smith et al., 1995; Williams, Weinmman, y Dale, 1998) comprueban que la primera razón por la cual los pacientes se declaran satisfechos de la atención recibida, tanto en hospitales como en centros de salud, es el trato humano que reciben por parte del profesional sanitario que les atiende, destacándose como elementos fundamentales de este trato la empatía mostrada por los profesionales, la información comprensible que éstos aportan, el tiempo que dedican a la relación personal con el enfermo y la posibilidad que el paciente tiene de expresarse.

Así pues, la comunicación humana es un proceso de intercambio de información entre un emisor y un receptor, que supone la percepción de un significado común entre ambas partes (Musitu, 1996). La comunicación es un elemento esencial en el funcionamiento social humano, ya que permite a los individuos y los grupos relacionarse entre sí, conformando de este modo, la misma sociedad. En todos los aspectos esenciales de las organizaciones laborales interviene la comunicación humana de una manera fundamental (Peiró, 1998). Esta manera de entender la comunicación humana lo aportaron los enfoques relacionales representados por la Escuela de Palo Alto con autores como Bateson, Birdwhistell y Watzlawick, haciendo explícitos los axiomas de la comunicación humana y permitiendo acercarse a la comunicación desde la complejidad de las relaciones humanas (Pinazo y Pastor, 2006; Winkin, 2008).

Los profesionales de la salud que trabajan con pacientes que han sufrido una amputación traumática, se ven con frecuencia expuestos a situaciones complejas en 
las que la comunicación va a ser imprescindible para su manejo.

Se calcula que aproximadamente el 1,8 por mil de la población española son amputados (Instituto Nacional de Estadística, 2008). En la región de Murcia la tasa de población con amputaciones/agenesia está en torno al 1,2 por mil. La mayoría por enfermedades degenerativas asociadas al envejecimiento, la enfermedad isquémica, la diabetes, etc. Los traumatismos suponen la séptima causa de amputación. Dentro de los traumatismos encabezan el ranking los accidentes de tráfico $(36,75 \%)$, seguidos de otros accidentes (35\%), complicaciones de la cirugía $(5,42 \%)$ y otras causas en menor porcentaje, como por ejemplo accidentes ferroviarios, suicidios y caídas accidentales (Instituto Nacional de Estadística, 2004). En Estados Unidos se calcula que una de cada doscientas personas ha sufrido algún tipo de amputación, siendo también las más frecuentes las asociadas a enfermedades crónicas y vasculares (Dillingham, 2002). En nuestro contexto encontrar una persona joven con una amputación de miembros inferiores no es tarea fácil. Se calcula que en España hay una tasa de amputados entre 6 y 24 años del 0,31 por mil (Instituto Nacional de Estadística, 2008).

Las amputaciones traumáticas tienen su importancia no por su elevada prevalencia sino por suponer un cambio desestructurante en las vidas de los sujetos que las padecen y sus familias. Cada vez resulta más difícil ver personas amputadas. Los avances en la prevención de enfermedades degenerativas y la disminución de accidentes laborales y de tráfico ha reducido considerablemente el número de personas a las que les falta una pierna. Sobre todo es complicado ver, afortunadamente, personas jóvenes con este problema. No obstante, en nuestro entorno conviven personas amputadas de miembros inferiores cuyos testimonios acerca de su itinerario de padecimiento hemos recogido aquí.

Todas las amputaciones no son iguales. Las amputaciones traumáticas tienen sus particularidades. En el caso de las personas que padecen una enfermedad vascular concurren diversos factores que hacen que la vivencia de la amputación sea muy distinta a los que pierden una pierna de forma traumática. El primero de ellos es la edad, en muchas ocasiones avanzada. Las personas de más de cincuenta años representan la mayoría de los amputados vasculares, mientras que los amputados jóvenes lo son a consecuencia de accidentes o por el desarrollo de tumores (Institut Desvern, 2003). Las expectativas, los proyectos de vida y las posibilidades -teóricamente- son más reducidas que en los jóvenes.

El sentido otorgado a la intervención es muy diferente. En uno liberador, en otro carente de sentido. En aquellos que padecen una enfermedad vascular crónica y degenerativa que implica experimentar dolor a diario "el anuncio de la amputación puede despertar expectativas de disminución del sufrimiento", mientras que en las amputaciones accidentales "la vivencia puede ser muy traumática y puede ir acompañada de una ansiedad intensa en la que se recuerda el accidente de forma reiterada" (Cañete, 2008: 479). En la mayoría de los amputados traumáticos, como hemos visto, se atribuye a la casualidad, al azar, al capricho otorgándose un sentido negativo a la génesis de la situación a la que se ven abocados. 
El poder de la palabra en la gestión del sufrimiento en el contexto hospitalario es un hecho que pasa necesariamente por una comunicación terapéutica entre el personal sanitario (que conoce, maneja el padecimiento y decide) y el que sufre. Según Hernández (2010: 23):

"La experiencia nos confirma que las palabras atenúan los dolores del cuerpo y mitigan los sufrimientos del espíritu, suavizan los angustiosos interrogantes y poseen la capacidad de hacernos sentir menos solos. (...) Durante las enfermedades -quizás más que en otras situaciones- nos viene muy bien la compañía de hombres y mujeres que, desinteresados, libres, equilibrados y prudentes, sin pedirnos nada a cambio, nos ayuden a dominar el horror ante lo desconocido, transmitiéndonos mensajes tranquilizadores: que nos cuenten con delicadeza, con objetividad y con generosidad, los riesgos que nos acechan, sin aprovecharse de nuestros temores".

Dentro de esa facultad que tiene el lenguaje de transmitir explicaciones acerca de cuestiones que interesan en la recuperación del enfermo, la administración de la información es de crucial importancia. Tradicionalmente se ha asociado el manejo de la información con el poder y la dominación de los que saben (carácter cuasi sagrado) sobre los profanos. La Medicina institucionalizada ha manejado la información científica y técnica como modo de control social, mostrando su hegemonía fundada en saberes sobre otras prácticas curativas o de atención (Good, 2003). De la gestión arbitraria de la información se deriva precisamente gran parte del malestar que perciben los amputados.

Uno de los principales escollos a los que se enfrenta el amputado es la falta de información. No sólo en nuestro contexto. Estudios realizados en lugares como Sudáfrica contemplan la falta de información como uno de los factores que acrecientan el sufrimiento de estas personas en ese contexto, proponiendo técnicas como la "imaginación guiada" (guided imagery) para mejorar los programas de rehabilitación (Mountany, 2007). El manejo precario de la información se hace más patente en los amputados traumáticos. Las propias características de su proceso, calificado como emergencia, gravedad, crítico, catastrófico, etc., hacen que incluso la decisión de amputar no se les comunique ya que en muchas ocasiones se encuentran sedados o anestesiados.

Comunicar malas noticias es una frecuente y difícil tarea con la que tienen que enfrentarse los profesionales sanitarios, a pesar de lo cual ha sido considerada como una competencia menor comparada con los otros aspectos técnicos de la práctica médica, y para lo que no se ha previsto una formación específica de comunicación en los planes curriculares, tanto de pre como de posgrado (García, 2006; Núñez, Marco, Burillo-Putze y Ojeda, 2006).

Muchos de los profesionales que tratan con pacientes creen que deberían dar sólo buenas noticias y que, si no hay ninguna, es mejor no decir nada. Puede que ello facilite el trabajo del médico, puesto que dar malas noticias es un asunto difícil y, a menudo, emocional. Sin embargo, para el paciente, "ninguna noticia" significa "no buenas noticias", y eso es una invitación al miedo. 
Así pues, la falta de información puede ser problemática, de la misma manera que una información clara facilita la buena comunicación. La falta de información genera incertidumbre y ésta genera ansiedad, depresión y miedo (Rodríguez-Marín y Neipp, 2008). Por otra parte, existen diversas razones legales que apoyan la idea de que informar a los enfermos es un hecho incuestionable. La obligación legal surge a partir de los nuevos códigos que rigen las relaciones médico-pacientes y que obligan a que éstas se guíen por los conocidos consentimientos informados. En España, el artículo 10 de la Ley General de Sanidad (1986) expone que "el paciente tiene derecho a que se le dé, en términos comprensibles, a él y sus familiares, información completa y continuada, verbal y escrita sobre su proceso, incluyendo diagnóstico, pronóstico y alternativas de tratamiento". Además, la Ley de Autonomía del Paciente y de Derechos y Obligaciones en Materia de Información y Documentación Clínica (2004), en su capítulo II, recoge el derecho de los pacientes a la información sanitaria.

Por tanto, adentrarse en la subjetividad de las personas amputadas por accidente nos parece conveniente. Así, los discursos referidos a la esfera de la comunicación informativa entre el personal sanitario y los amputados, sobre todo, en los momentos que rodean el acto quirúrgico o la pérdida del miembro en el accidente, son vitales para conocer las circunstancias y características que acompañan a este proceso.

\section{Objetivos}

Como objetivos en este estudio, nos planteamos:

- Conocer la valoración subjetiva de la atención recibida durante el ingreso hospitalario de las personas que han sufrido una amputación de miembro inferior en accidente de moto.

- Analizar las opiniones en torno a la comunicación informativa ofrecida a los recién amputados por causa traumática.

\section{Metodología}

Este estudio de corte cualitativo se ha realizado usando la etnografía como principal método de investigación. Se escogió como unidad de análisis principal a personas amputadas de miembros inferiores. La muestra principal la han constituido diecinueve amputados.

También se han escogido otros informantes de entre las personas que rodean al amputado y de entre las que se han involucrado en la gestión de su padecimiento. En especial familiares y amigos. Asimismo entrevistamos a profesionales directamente implicados en la gestión de su problema (ortopedas, fisioterapeutas y médicos traumatólogos).

Se siguió una forma de muestreo conocida como en "bola de nieve" o de avalancha (Polit y Hunger, 2000). Es una forma de muestreo no probabilístico usado con frecuencia en investigación cualitativa y concretamente en el método etnográfico. Consiste en escoger a uno o varios informantes y solicitarles que, a su vez, recomienden a otras personas a participar. También se le conoce como muestreo en cadena. Partiendo 
de unos cuantos informantes claves se consiguió contactar con otros que a su vez facilitaban el acceso a nuevos entrevistados. Uno de los "porteros" más importantes fue el tercer informante (E.A.4). Una asociación de discapacitados a nivel regional (FAMDIF) proporcionó el contacto. Gracias a él se conseguió entrevistar a cuatro o cinco personas, que a su vez derivaron a otras con las características o criterios de inclusión que establecidos. Por acotar y centrar la población de estudio, se creyó conveniente seleccionar exclusivamente amputados de miembros inferiores a cualquier nivel, tibial o femoral (movilidad aún más limitada), de una o ambas piernas. El tipo de amputación (traumática o no) fue también uno de los criterios tenidos en cuenta a la hora de seleccionar la muestra.

Se destaca la relación de la mayoría de los informantes con el mundo de las motos. En el total de 19 entrevistados, la mayoría de los amputados (14) lo son por accidente de moto, uno por accidente de coche y cuatro por enfermedad. La mayoría de los informantes amputados son adultos de mediana edad. En cuanto al tiempo transcurrido desde la amputación, la mayor parte hacía más de dos años que había sufrido el accidente o la intervención. Existen, no obstante, testimonios de personas recién amputadas.

Se han recogido los datos valiéndose de la entrevista semiestructurada, el método biográfico y la observación participante. Las entrevistas se realizaron a sujetos que habían sufrido la amputación de una o las dos piernas. Se recogió información de aspectos referidos a las categorías que nos interesaba estudiar. Previamente a la realización de la entrevista se elaboró un guión general de las cuestiones a tratar.

Respecto al método biográfico, ofreció la perspectiva que el tiempo otorga a las circunstancias vividas por los sujetos. En nuestro caso los contenidos no se ajustaban a la totalidad de una biografía sino más bien se restringían a ciertos contenidos temáticos.

Se recogieron también documentos personales, audiovisuales y escritos, que han servido para complementar y enriquecer el discurso de los informantes. Una vez transcritas las entrevistas, se introducían en un programa de codificación y análisis cualitativo, el MAXQDA2@. A la hora de analizar los datos se ha seguido el estilo etnográfico de Taylor y Bogdan (1994), que describen tres fases: descubrimiento, codificación y relativización.

\section{Resultados y discusión}

Los informantes describen el tiempo transcurrido en su ingreso hospitalario como un periodo ventana entre el hecho traumático y la vuelta a la realidad. Los testimonios hablan de estar flotando, como en una nube, sin ser conscientes (incluso durante meses) de lo que implicaba poseer un cuerpo en cierto modo incompleto. Según Arthur Kleinman, las instituciones encargadas de prestar los cuidados "son a menudo en sí mismas productoras de sentimientos de amenaza y desamparo, como cuando el dolor o el sufrimiento no se valoran o cuando las peculiares formas de experimentar la enfermedad se diluyen en categorías diagnósticas" (Kleinman, 2004: 
18). En este sentido veremos cómo la gestión inicial del sufrimiento del amputado pasa ineluctablemente por este lugar de paradoja: donde se alivia el sufrimiento es donde también, en ocasiones, se promueve.

Según los protocolos de actuación en el paciente amputado, la ayuda psicológica debe solicitarse durante el postoperatorio "si se presenta la necesidad" (Serra, 2001). De los testimonios obtenidos, se infiere que el apoyo psicológico profesional en las personas que hemos entrevistado ha sido escaso o nulo. También ha contribuido al malestar y al sufrimiento de estas personas la falta de información clara, sobre todo en los casos en los que se atisban errores o negligencias percibidas como tales por los usuarios de las instituciones sanitarias. Las diferentes visiones del problema, la escasez de casos similares, la compartimentación del ser humano en cuerpo/mente, con la consiguiente preeminencia de los cuidados corporales, lo hacen difícil. En otros casos no se ha demandado una asistencia psicológica y, si ésta se ha ofrecido, algunos sujetos la han rechazado. Lo que querían era irse a casa cuanto antes. Al siguiente informante le parece ridículo el apoyo psicológico recibido, él quería irse a su casa:

“...Y luego la psicóloga también parecía que no sé, que tenía doce años o algo así... ¿quieres unos puzles o unos cuentos o algo? Estaba mi madre delante ¿esto qué es? Si, si es que estas cosas la verdad es que..." (E.10.A).

Cuando no se puede salvar el miembro se va intuyendo lo que va a suceder. En el siguiente relato, nuestro entrevistado sabía que el final de su pie iba a ser la amputación. Ante nuestra pregunta de si recibió la visita de un profesional que le preparase para la amputación afirma:

“...Sí, pero, hombre tonto no soy. Yo sabía que aquel pie lo tenían que amputar. Cada día estaba más negro, parecía una hamburguesa aplastada contra una barbacoa (...) los últimos días entró el psiquiatra o el psicólogo, pero a esas alturas, que venía más o menos a prepararme, yo ya lo sabía. Lo sabía por intuición propia" (E.11.A).

Otro de los informantes evoca en su memoria aquella etapa. Estaba como en una nube, habla de este periodo y coincide con que los cuidados y la atención en casi todos los aspectos impiden darse cuenta de que no iba a ser fácil. La psicóloga del hospital le ayudó a "poner los pies en el suelo":

“...El proceso ese de hospitalización fue bueno, mi mujer fue la única que se dio cuenta de que yo estaba demasiado entusiasmado (...) Yo pensaba que iba a trabajar en veinte días, que yo iba a estar igual que antes (...) yo no me daba cuenta realmente de lo que tenía, y mi mujer sí, y me hizo hablar con la psicóloga del hospital, y ella me ayudó un poquito (...) y ya me puso un poquito los pies en el suelo." (E.12.A).

El traumatólogo sentencia que en estos casos el papel del paciente en la toma de decisiones es cero. Cuando abundamos en la pregunta de si se prepara psicológicamente al paciente para lo que se le viene encima la respuesta es: 
“...Al psicólogo no te da tiempo. Luego en planta, depende de cómo tú veas cómo lo acepta sí que llamas...aquí en el hospital no hay psicólogo. Aquí el psiquiatra hace de psiquiatra y psicólogo ¿no? Entonces se habla con el paciente cuando se despierta..." (E.1.T).

Salvar el miembro supone la segunda prioridad tras salvar la vida. Aquellos que sufren complicaciones como la gangrena a veces se someten al tratamiento en cámaras hiperbáricas precisamente para poder salvar la pierna o en todo caso que el nivel de la amputación sea lo más bajo posible. Es algo que determinará la vida posterior y la independencia en gran medida (Serra, 2001). Conservar la rodilla supone un mundo. Un informante, doble amputado femoral, considera que a la hora de tomar la decisión de amputar no le informaron de la posibilidad de aguantar más tiempo con el tratamiento para poder salvar la rodilla de una de sus piernas:

“...Si hubiera sabido la diferencia que suponía el desenvolverse con una rodilla me hubiera esperado allí no dos meses, dos años dentro de la cámara hasta que aquello se hubiera solucionado (...) ¡Me malinformaron! Porque, ¡claro!, yo estaba ocupando una habitación ya un mes ahí, ¿me entiendes? Una habitación que era de estar solo (...) En el momento que tienes una rodilla es otro mundo...y si tienes las dos no te digo (...) Como yo voy es lo más difícil que hay, porque es que andas con la cintura." (E.3.A).

En otras ocasiones la temática es tan tabú que se espera hasta transcurridos unos días para hablar del tema con el afectado (que ya intuye lo que tiene o mejor dicho, lo que le falta). A veces, el manejo de la información se gestiona con un sentido protector, sobre todo en los menores de edad. Para Goffman (2001), el control de la información sobre el estigma en los niños que tienen alguna discapacidad, pasa por un periodo de ocultamiento y sobreprotección. El control de la información contribuye a que se encapsule el problema. Goffman (2001) denomina individuo protegido precisamente al estigmatizado cuya familia y el entorno han obviado cierta información sobre su falta. A una persona que entrevistamos le atropella una moto con 14 años, le duelen las dos piernas, tiene sensaciones en ambas extremidades aunque le acaban de amputar una. Nadie se lo comunica. Nadie se atrevió a decírselo. Le ponen una sábana por encima. Sólo una monja se acerca y le cuenta:

“... ¿Qué me pasa en las piernas que no las puedo mover?, y nadie me decía nada, porque nadie se atrevía a decírmelo. Y yo decía: ¿por qué no las puedo mover? (...) Y me pusieron una sabana por encima (...) Entonces fue una monja (...) habló conmigo (...) que yo me iba a poner bien, y que me iban a poner una prótesis igual que mi pierna, que yo no iba a notar nada" (E.15. A).

Estar más de un mes ingresado desde que le atropellan hasta que le amputan es la experiencia de otro informante. Las curas son momentos horribles. Su versión es mala también. Dolor, curas, incertidumbre. Le van preparando el cuerpo, le van insinuando que la cosa puede terminar en amputación:

“...Me dicen que si no hay riego sanguíneo al final va a ser amputación. Pero que 
yo podía hacer esto (movimiento leve del pie), yo intentaba moverlo todo lo que podía, todo el tiempo así, 24 horas al día. Se movía un poquico, pero eso iba cada vez más negro y más negro..." (E.18. A).

Algunos dan cuenta de esa sensación experimentada de levantarse sin pierna. El siguiente informante no sabe que existen prótesis, nadie le informa. Le amputan la pierna y lo primero que se le viene a la cabeza es "voy a ser un estorbo".

“...Cuando me levanté de la cama, una sensación muy rara. Que estás ahí, en un punto solo. Apoyado con un pie, que no puedes hacer nada, nada más que estar de pie. Yo al principio es que no sabía que había prótesis ni nada (...) No tenía información ninguna (...) se te cae el mundo (...) vas a ser un estorbo para todo el mundo." (E.14. A).

A este otro, también menor de edad, no le informan de que le van a cortar la pierna. Luego, le argumentan que era mejor así.

“...A mí no me dijeron, pero dicen... jes que es mejor! Bueno, si es mejor, vosotros sabéis lo que hacéis. Yo estoy aquí vivo y ya está. Sobran palabras." (E.7. A).

En un caso, a los días de haber sufrido la amputación le dan oficialmente la noticia que ya sabía. Constatamos que la información tardía (mal administrada) tiene poco efecto terapéutico en esta persona.

"...Me acuerdo que entraron también unas médicas a decirme que me habían amputado una pierna, que me pareció una gilipollez (...) Has tenido un accidente, te hemos tenido que amputar, me pareció una tontería." (E.11.A).

La práctica médica tiene en la infalibilidad uno de sus pilares. El método científico augura que ante los mismos procedimientos, elementos y circunstancias se pueden reproducir los resultados de manera idéntica. Operar con el cuerpo como elemento estático o mecánico, sometido a las mismas leyes de la naturaleza, que responde bajo unos parámetros universales. Es ese saber el que se maneja y el que maniobra en las conciencias de los que acuden y se someten a la disciplina científica confiando en que no hay lugar para la incertidumbre. Asumir el carácter variable de la práctica asistencial quitaría un peso de encima a profesionales y usuarios. Someterse al cuidado y a la reparación supone, en cierto modo, emprender un viaje incierto del mismo modo en el que los antiguos se embarcaban rumbo a lo desconocido, sometidos a la contingencia. Por eso imploraban a los dioses, consultaban oráculos y recitaban plegarias. Por la incertidumbre. Autores como el filósofo José Luis González Quirós (2008), opinan que la Medicina tiene que asumir que la gestión de la incertidumbre tiene que apartarse de los planteamientos taxativos que su condición de ciencia le determina de manera encorsetada. Sería preciso ver la incertidumbre como la regla y no como la excepción. De ese modo no se crearían falsas expectativas y se dejaría paso a una práctica médica más relajada, a una información más abierta y más realista:

"El médico tiene que aprender a gestionar la incertidumbre, muchos niveles de 
ambivalencia, de riesgo y de carencias esenciales de información. ¿Cómo se puede aprender a gestionar la incertidumbre? La única receta que se me ocurre es la de dejar de ver la incertidumbre como una excepción, aprendiendo a ver que esa es la situación normal, sino en la totalidad, sí en la mayoría de los casos. No cabe duda que eso exige una formación hermenéutica que permita tener una visión de los saberes más ajustada a su efectiva realidad que la que proporciona una imagen cientifista del mundo". (González, 2008: 12).

En este sentido es pertinente apuntar aquí las ideas que como marco conceptual en salud se proponen desde el modelo salutogénico (Rivera, Ramos, Moreno y Hernán, 2011). Al asumir el propio individuo las riendas del manejo de su salud, el papel del profesional sanitario se fundamentaría en el apoyo y el ofrecimiento de opciones para que la toma de decisiones sea tarea del sujeto en un diálogo recíproco sin paternalismos ni posiciones hegemónicas. Ciertamente la idea patogénica de la vida contrastaría con la salutogénica, fomentando ésta última la constante recreación de la salud sin considerar que ésta es un bien continuo y omnipresente, sino más bien una parte del proceso vital donde coexisten desequilibrio, dolor, enfermedad, sufrimiento con momentos de bienestar y salud.

Hacer ver esto tanto a los pacientes como a los profesionales no es tarea fácil, pero este modelo, poco a poco, se abre camino en la planificación de las políticas de salud y requiere precisamente de la potenciación de los propios recursos generales de resistencia de la persona (Rivera et al., 2011), y esto sin duda se fundamenta en una visión dinámica del binomio salud/enfermedad que ha de materializarse mediante mecanismos que incluyan una adecuada comunicación que abra una vía a la esperanza, alejando al amputado de una visión patogénica de su proceso.

En el ámbito latinoamericano, existen publicaciones que hacen hincapié en la necesidad de informar correctamente a los pacientes que van a ser amputados, cosa que en la práctica no se realiza de forma efectiva: "Es frecuente que éste dé su consentimiento para someterse a un tratamiento, sin que haya tenido una correcta comunicación con el médico que va a realizarlo" (Artaza, 2005: 5). De hecho, como expresan RodríguezMarín y Neipp (2008), para el paciente "ninguna noticia" significa "no buenas noticias", y eso es una invitación al miedo, por lo que la falta de información genera incertidumbre y ésta genera ansiedad, depresión y miedo.

Otros autores destacan la importancia de un abordaje multidisciplinar en la comunicación de malas noticias (Mirón, 2010) y que los profesionales de la salud deberían poseer unas adecuadas habilidades sociales y de comunicación para hacer frente a este tipo de situaciones (Leal, Luján, Gascón, Ferrer y van-der Hofstadt, 2010). Ya hemos visto que, en la práctica, las personas amputadas que hemos entrevistado reclaman un mayor énfasis en la información y la gestión más humana de su padecimiento. Comunicar peor significa generar más sufrimiento.

\section{Conclusiones}

En el hospital al principio todo es confuso. En el amputado por accidente es un 
periodo casi onírico. No se es plenamente consciente del problema. No se acaba de interiorizar que falta una parte. Los familiares perciben (sufren) la verdadera dimensión de lo ocurrido. El hospital es percibido como un lugar de sufrimiento y de esperanza. La hospitalización es un periodo ventana que conlleva una euforia inicial. Se prestan cuidados y atenciones que, aunque necesarios, enmascaran la falta ("uno está como flotando"). Desaparecen signos que confieren identidad. Rutinas terapéuticas marcan el ritmo. Otros deciden por uno. La información se gestiona por aquellos que poseen el saber técnico.

La información se dosifica y se gestiona de forma precaria en la mayoría de los casos, se informa de forma tardía en los pacientes amputados traumáticos. No se da importancia al hecho de que el individuo y su familia estén puntual y correctamente informados. No se observan señales de comunicación terapéutica en la mayoría de los casos. El manejo de la información sigue siendo una moneda de cambio que mantiene las estructuras de poder en el ámbito sanitario. La desinformación provoca malestar y contribuye a aumentar el sufrimiento de los amputados. Esto es más evidente en los amputados traumáticos. La amputación, en algunos casos, fue un tema tabú que nadie se atrevía a tratar, sobre todo en jóvenes. El apoyo psicológico profesional ha sido escaso o nulo en el periodo de hospitalización. Existe, bajo el punto de vista de los entrevistados, una falta de información clara.

Como líneas a considerar en futuras investigaciones e intervenciones, hemos de argumentar la necesidad de configurar herramientas que sirvan de forma práctica tanto a los profesionales como a los pacientes para entender y superar una amputación lejos del victimismo y con una visión realista y a la vez compasiva de la situación de sufrimiento por la que temporalmente discurre la vida de los amputados. En este sentido, se está elaborando un manual para amputados y profesionales, desarrollado por un equipo multidisciplinar de autores (enfermeros, antropólogos y psicólogos) y que se fundamenta en esta investigación, que podría dar respuesta a las cuestiones que aquí se tratan.

\section{$\overline{\text { Referencias }}$}

Artaza Sanz, Heriberto M. y Gómez Martínez, Marxlenin. (2005). La bioética en el manejo del paciente amputado de los miembros inferiores y de sus familiares. Revista Habanera de Ciencias Médicas. Vol. 4, nº 5, pp. 1-9.

Bredart,Anne;Bouleuc, Caroley Dolbeault, Sylvie.(2005). Doctor-Patient Communication and Satisfaction with care in Oncology. Current Opinion in Oncology. Vol. 17, pp. 351354.

Cañete Crespillo, José y Cañizares Alejos, Silvia. (2008). Aspectos psicológicos y psiquiátricos del paciente amputado. En Alós Villacrosa, Jesús (Ed.). Amputaciones del miembro inferior en cirugía vascular (pp. 475-496). Barcelona: Glosa.

Dillingham, Timothy R.; Pezzin, Liliana E. y MacKenzie, Ellen J. (2002). Limb Amputation 
and Limb Deficiency: Epidemiology and Recent Trends in the United States. Southern Medical Journal. Vol. 95, pp. 875-883.

García Díaz, Fernando. (2006). Comunicando malas noticias en medicina: recomendaciones para hacer de la necesidad virtud. Medicina Intensiva. Vol. 30, no 9, pp. $452-459$.

General Medical Council. (2001). Good Medical Practice. London: General Medical Council.

Goffman, Erving. (2001). Estigma. La identidad deteriorada. Buenos Aires: Amorrortu.

González Quirós, José Luis. (2008). Medicina, antropología y política en la formación del médico. Simposio Internacional sobre valores, hechos y razones en el ámbito sanitario: la racionalidad acotada. Madrid: Universidad Autónoma de Madrid.

Good, Byron J. (2003). Medicina, racionalidad y experiencia. Una perspectiva antropológica. Barcelona: Bellaterra.

Hernández, J.A. (2010). ¿Curan las palabras? Manual de comunicación médica y sanitaria. Alicante: Biblioteca virtual Miguel de Cervantes.

Institut Desvern de Protética (2003). Manual para amputados de extremidad inferior. Barcelona: Institut Desvern.

Instituto Nacional de Estadística (INE). (2004). Registro de Altas de Hospitalización entre los años 2000 y 2004.

Instituto Nacional de Estadística (INE). (2008). Encuesta de Discapacidad, Autonomía Personal y Situaciones de Dependencia 2008 Discapacidades, Deficiencias y Estado de Salud. Resultados nacionales: cifras relativas.

Kleinman, Arthur. y Benson, Peter. (2004). La vida moral de los que sufren enfermedad y el fracaso existencial de la medicina. En Bayés, Ramón (Ed.). Dolor y sufrimiento en la práctica clínica (pp. 17-26). Barcelona: Fundación Medicina y Humanidades Médicas.

Lázaro, José y Gracia, Diego. (2006). La relación médico-enfermo a través de la historia. Anales del Sistema Sanitario de Navarra. Vol. 29, Supl. 3, pp. 7-17. DOI 10.4321/S1137-66272006000600002.

Leal Costa, César, Luján Cebrián, Isabel, Gascón García, Josefa, Ferrer Villalonga, Lorenzo y van-der Hofstadt Román, Carlos Javier. (2010). Habilidades sociales en los profesionales de urgencias y cuidados críticos de un hospital público comarcal. Enfermería Intensiva. Vol. 21, nº 4, pp. 136-141. DOI 10.1016/j.enfi.2010.05.001.

Ley 41/2002 de 14 de noviembre, Básica Reguladora de la Autonomía del Paciente 
y de Derechos y Obligaciones en Materia de Información y Documentación Clínica. España.

Ley 14/1986 General de Sanidad, de 25 de abril. España

Mead, Nicola y Bower, Peter. (2000). Patient-Centredness: A Conceptual Framework and Review of the Empirical Literature. Social Science \& Medicine. Vol. 51, pp. 1.0871.110 .

Mira, José Joaquín y Aranaz, Jesús. (2000). La satisfacción del paciente como una medida del resultado de la atención sanitaria. Medicina Clínica. Vol. 114, Supl. 3, pp. 26-33.

Mirón González, Rubén. (2010). Comunicación de malas noticias: perspectiva enfermera. Revista Española de Comunicación en Salud. Vol. 1, n 1, pp. 39-49.

Mountany, Rochelle. (2007). The Experience of Self of the Amputee: An Ecosystemic Investigation. Tesis doctoral. South Africa: University of South Africa.

Musitu Ochoa, Gonzalo. (1996). Manual de psicología de la comunicación. Valencia: Editorial Cristóbal Serrano Villalba.

Núñez, Salvador; Marco, Teresa; Burillo-Putze, Guillermo y Ojeda, Juan. (2006). Procedimientos y habilidades para la comunicación de las malas noticias en urgencias. Medicina Clínica. Vol. 127, nº 15, pp. 580-583.

Peiró, José María. (1998). Psicología de la organización. Madrid: Universidad Nacional de Educación a Distancia.

Pinazo Hernandis, Sacramento y Pastor Ruiz, Yolanda. (2006). Modelos teóricos en el estudio de la comunicación. En Yolanda Pastor Ruiz (Coord.). Psicología Social de la Comunicación (pp. 19-43). Madrid: Ediciones Pirámide.

Polit, Denisse F. y Hunger, Bernardette P. (2000). Diseños de muestreo. En Polit, Denisse F. y Hunger, Bernardette P. Investigación científica en ciencias de la salud (pp. 285-289). México: McGraw-Hill,

Rivera de los Santos, Francisco; Ramos Valverde, Pilar; Moreno Rodríguez, Carmen y Hernán García, Mariano. (2011). Análisis del modelo salutogénico en España: aplicación en salud pública e implicaciones para el modelo de activos en salud. Revista Española de Salud Pública. Vol. 85, pp. 129-139

Rodríguez-Marín, Jesús y Neipp López, Ma Carmen. (2008). Manual de Psicología Social y de la Salud. Madrid: Síntesis

Serra Gabriel, Ma Rosa. (2001). El paciente amputado. Labor de equipo. Barcelona: Springer. 
Simón Lorda, Pablo. (2000). El consentimiento informado. Madrid. Triacastela.

Smith, Robert C. et al. (1995). A Strategy for Improving Patient Satisfaction by the Intensive Training of Residents in Psychosocial Medicine: A Controlled, Randomized Study. Academic Medicine. Vol. 70, pp. 729-732.

Taylor, Steve J. y Bogdan, Robert. (1994). Introducción a los métodos cualitativos de investigación. Barcelona: Paidós.

WHO. (1993). Doctor Patient Interaction and Communication. Geneva: World Health Organization, Division of Mental Health. Report N WHO-MNS-PSF-93.11.

Winkin, Yves. (2008). La nueva comunicación. Barcelona: Kairós.

Williams, S.; Weinmman, J. y Dale J. (1998). Doctor-Patient Communication and Patient Satisfaction: A Review. Family Practice. Vol. 15, pp. 480-492. 